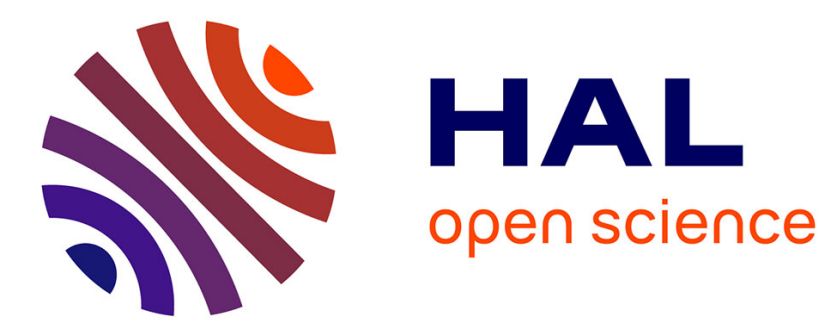

\title{
Tensorial rheological model for concentrated non-colloidal suspensions: normal-stress differences
}

Olivier Ozenda, Pierre Saramito, Guillaume Chambon

\section{To cite this version:}

Olivier Ozenda, Pierre Saramito, Guillaume Chambon. Tensorial rheological model for concentrated non-colloidal suspensions: normal-stress differences. Journal of Fluid Mechanics Digital Archive, 2020, 898, pp.A25. 10.1017/jfm.2020.405 . hal-02138682v3

\section{HAL Id: hal-02138682 \\ https://hal.science/hal-02138682v3}

Submitted on 18 May 2020

HAL is a multi-disciplinary open access archive for the deposit and dissemination of scientific research documents, whether they are published or not. The documents may come from teaching and research institutions in France or abroad, or from public or private research centers.
L'archive ouverte pluridisciplinaire HAL, est destinée au dépôt et à la diffusion de documents scientifiques de niveau recherche, publiés ou non, émanant des établissements d'enseignement et de recherche français ou étrangers, des laboratoires publics ou privés. 


\title{
Tensorial rheological model for concentrated non-colloidal suspensions: normal-stress differences
}

\author{
Olivier Ozenda ${ }^{1,2}$ Pierre Saramito $^{1} \dagger$ and Guillaume Chambon ${ }^{2}$ \\ ${ }^{1}$ Lab. J. Kuntzmann - CNRS and Univ. Grenoble Alpes, CS 40700 - 38058 Grenoble Cedex 9, \\ France \\ ${ }^{2}$ Univ. Grenoble Alpes, IRSTEA, UR ETGR, 2 rue de la Papeterie, 38402 St-Martin-d'Hères, \\ France
}

(Received xx; revised xx; accepted xx)

Only few rheological models in the literature simultaneously capture the two main nonNewtonian trends of non-colloidal suspensions, namely finite normal stress differences and transient effects. We address this issue by extending a previously-proposed minimal model accounting for microstructure anisotropy through a conformation tensor, which was shown to correctly predict transient effects (Ozenda et al. 2018). A systematic sensitivity study was performed to provide insights into the physical interpretation of the different model terms. This new model is compared to a large experimental dataset involving varying volume fractions, from dilute to concentrated cases. Both apparent viscosity and normal stress differences in steady-state, are quantitatively reproduced in the whole range of volume fraction, and qualitative agreement for transient evolution of apparent viscosity during shear-reversal is obtained. Furthermore, the model is validated against particle pressure measurements that were not used for parameter identification. Even if the proposed constitutive equation for the Cauchy stress tensor is more difficult to interpret than in the minimal model, this study opens way for the use of conformation tensor rheological models in applications where the effect of normal stress differences is prominent, like elongational flows or particle migration processes.

\section{Introduction}

Concentrated suspensions of non-colloidal rigid particles present two main nonNewtonian rheological trends, namely finite normal stress differences under shear flow and transient effects (see, e.g., the recent review by Guazzelli \& Pouliquen 2018). (i) Since the early work of Gadala-Maria (1979), normal stress differences have been investigated in different flow geometries (Zarraga et al. 2000; Singh \& Nott 2003; Couturier et al. 2011; Boyer et al. 2011; Dai et al. 2013; Dbouk et al. 2013). These measurements consistently show that the second normal stress difference $N_{2}$ is negative, with an absolute value that grows with volume fraction $\phi$. The first normal stress difference $N_{1}$ is generally found to be much smaller, in absolute value, than $\left|N_{2}\right|$. While most studies reported negative $N_{1}$ values, few also reported positive values especially at high volume fractions. (ii) Using shear-reversal experiments, Gadala-Maria \& Acrivos (1980) showed that non-colloidal

$\dagger$ Email address for correspondence: pierre.saramito@math.cnrs.fr 
suspensions present transient rheological responses, characterized by a rate-independent evolution of the shear viscosity over typical strains of a few units, before this quantity eventually reaches its steady-state value. These shear-reversal experiments were later revisited by Kolli et al. (2002) and Blanc et al. (2011). All these authors related the transient effects to the development of microstructure anisotropy under shear flow, and attempted to quantify this anisotropy using the pair distribution function.

Most non-Newtonian trends of concentrated suspensions can be captured by particlebased numerical simulations, which offer additional insights into the physical processes at play. Earliest approaches were based on Stokesian dynamics, in which hydrodynamic interactions are split into far-field and near-field (lubrication) contributions (Brady \& Bossis 1985). Numerous studies showed that essential ingredients to obtain realistic rheological behaviour in these simulations have to be added, in particular, the existence of finite normal-stress differences, is to account for particle contacts through shortrange repulsive forces (Nott \& Brady 1994; Sierou \& Brady 2002). Numerical results also demonstrated that frictional interactions should be taken into account to recover correct orders of magnitude for these normal-stress differences, i.e. $\left|N_{1}\right|<\left|N_{2}\right|$ (Sierou \& Brady 2002; Mari et al. 2014). Similar conclusions concerning the critical role of particle friction were reached by Gallier et al. (2014), using a more accurate DNS method to model hydrodynamic interactions. More recently Peters et al. (2016) and Chacko et al. (2018) were also able to successfully simulate transient effects during shear-reversal using DNS and simplified Stokesian dynamics approaches, respectively. These particlebased simulations make it possible to continuously vary physical parameters and perform numerical experiments, thereby providing datasets that are well complementary to "real" experiments. However, due to the high demand in computing power, they are still limited to small systems with a relatively low number of particles (a few thousands at most).

Unlike discrete particle-based models, continuous rheological models are suited to the simulation of large systems. However, the elaboration of constitutive laws able to reproduce both normal-stress differences and transient features has been proved a challenging task (e.g., Denn \& Morris 2014). General expressions for the bulk Cauchy stress tensor of suspensions can be obtained in the frame of two-phase mixture models (Jackson 2000; Nott et al. 2011; Baumgarten \& Kamrin 2019), and involve contributions from both the interstitial fluid and the particles. For practical applications, physical closures need to be postulated for these different contributions. Nott \& Brady (1994) introduced the suspension balance model (later extended by Morris \& Boulay 1999; Miller \& Morris 2006), which involves explicit empirical expressions for the particle-induced normal stress components. This model provides relatively accurate predictions for steadystate flows and, when coupled to an evolution equation for the volume fraction $\phi$ (that arises from mass and momentum conservation of the particle phase), can also account for particle migration processes. A similar type of approach based on explicit stress closures was recently followed by Singh et al. (2018), who proposed a model describing the transition to shear-thickening in highly-concentrated suspensions. By construction, however, these models do not attempt to capture transient rheological effects.

Transient effects can be described by introducing a conformation tensor, denoted $\boldsymbol{b}_{e}$ in this paper, which represents microstructure anisotropy. Note that conformation tensors are also sometimes referred to as texture tensors (see e.g. Lehoucq et al. 2015). For noncolloidal suspensions, the conformation tensor $\boldsymbol{b}_{e}$ needs to obey an evolution equation that ensures rate-independence, i.e. this equation should have no characteristic time (e.g., Goddard 1982). Hand (1962) formulated general properties for anisotropic fluids, 
and expressed the Cauchy stress tensor $\boldsymbol{\sigma}$ as a function of both $\boldsymbol{b}_{e}$ and the strain rate tensor $\dot{\gamma}$. Phan-Thien (1995) proposed a differential constitutive equation for the conformation tensor that led, for the first time, to predictions qualitatively in agreement with experimental observations during shear-reversal. Goddard (2006) revisited this approach, and proposed a model involving twelve material parameters and two tensors for describing the anisotropy. By a systematic fitting procedure on a limited dataset, he obtained numerical results in quantitative agreement with experiments for transient effects and normal stress differences. Stickel et al. (2006) proposed a simplified expression for the Cauchy stress tensor, which is linear in terms of both the strain rate and the conformation tensors (see also Stickel et al. 2007; Yapici et al. 2009). While this latter model involves thirteen free parameters, it failed to provide quantitative comparisons with shear-reversal experiments: an unexpected sharp spike in both apparent viscosity and normal stress differences was obtained at the time of the reversal. Using a much simpler model, involving only four free parameters and a linear evolution evolution for the conformation tensor, Ozenda et al. (2018) recently obtained a good quantitative agreement with the shear-reversal measurements of Blanc et al. (2011) for a wide range of volume fraction $\phi$. Furthermore, at the micro-structural scale, the model successfully reproduced both the pair distribution function and the depletion angles measured by Blanc et al. (2013). However, this simple model predicts unphysical normal stress differences.

The objective of this paper is to propose a continuous rheological model that provides quantitative predictions for both normal stress differences and transient effects, for dilute to concentrated suspensions. For that purpose, we revisit and extend the model proposed by Ozenda et al. (2018) based on the general expansion of Hand (1962). We limit consideration to rate-independent constitutive responses, i.e. to suspensions dominated by hydrodynamic (and possibly frictional) interactions. We also restrict attention to concentration ranges sufficiently below jamming, such that granular plasticity effects arising from sustained frictional contacts between particles (e.g. granular dilatancy, Pailha \& Pouliquen 2009; Baumgarten \& Kamrin 2019) do not come into play. In particular, our model does not include the shear-thinning and shear-thickening effects observed in more complex systems and/or close to jamming transition (see, e.g., Royer et al. 2016; Singh et al. 2018). When compared with previous tensorial approaches (Phan-Thien 1995; Goddard 2006; Stickel et al. 2006), the present model involves only seven adjustable parameters, and compares well with experiments. Section 2 presents the rheological model. Section 3 is devoted to simple shear flows. The system of time-dependent equations is expanded for this case, and the stationary solution is explicitly exhibited. The dependence of the material parameters upon volume fraction $\phi$ is investigated, based on asymptotic analyses in the dilute and highly concentrated limits. In section 4, model predictions are compared with a large number of experimental results regarding both transient effects and steady-state normal stresses. As the presented model involves seven dimensionless constants, a systematic sensivity study is performed in section 5. Finally, results are summarised and discussed in section 6 .

\section{Mathematical model}

\subsection{Problem statement}

Following Ozenda et al. (2018), let us introduce the conformation tensor $\boldsymbol{b}_{e}=d_{0}^{-2}\langle\boldsymbol{\ell} \otimes \boldsymbol{\ell}\rangle$, where $\boldsymbol{\ell}$ is the branch vector joining the centres of two neighbouring particles and $d_{0}$ is the 
average distance between neighbouring particle centers in an isotropic configuration at rest. In the isotropic configuration at rest, we have $\boldsymbol{b}_{e}=\boldsymbol{I}$, where $\boldsymbol{I}$ is the identity matrix. For convenience, we introduce the tensor $\gamma_{e}=c_{0}\left(\boldsymbol{b}_{e}-\boldsymbol{I}\right)$, where $c_{0}$ is a dimensionless constant that will be chosen later. The tensor $\gamma_{e}$ interprets as the deformation of the micro-structure with respect to rest configuration. As in Ozenda et al. (2018), we assume a linear evolution for $\gamma_{e}$ :

$$
\begin{gathered}
\frac{\mathscr{D}_{a} \gamma_{e}}{\mathscr{D} t}+\delta_{1}|\dot{\gamma}| \gamma_{e}-\dot{\gamma}=0, \\
\dot{\gamma}=\boldsymbol{\nabla} \boldsymbol{u}+\boldsymbol{\nabla} \boldsymbol{u}^{T}
\end{gathered}
$$

where $\dot{\gamma}$ is the strain rate tensor, $\boldsymbol{u}$ is the velocity field of the suspension, and $\delta_{1}$ is a positive dimensionless material parameter. The matrix norm $|\boldsymbol{\xi}|$ is defined as $|\boldsymbol{\xi}|^{2}=(\boldsymbol{\xi}: \boldsymbol{\xi}) / 2$ for any matrix $\boldsymbol{\xi}$, with $(\cdot: \cdot)$ denoting the double contracted matrix product. We use in $(2.1 a)$ a general Gordon-Schowalter tensor derivative (Gordon \& Schowalter 1972; Saramito 2016):

$$
\frac{\mathscr{D}_{a} \boldsymbol{\gamma}_{e}}{\mathscr{D} t}=\frac{\partial \boldsymbol{\gamma}_{e}}{\partial t}+(\boldsymbol{u} . \boldsymbol{\nabla}) \boldsymbol{\gamma}_{e}-W(\boldsymbol{u}) \boldsymbol{\gamma}_{e}+\boldsymbol{\gamma}_{e} W(\boldsymbol{u})-\frac{a}{2}\left(\dot{\boldsymbol{\gamma}} \boldsymbol{\gamma}_{e}+\boldsymbol{\gamma}_{e} \dot{\boldsymbol{\gamma}}\right)
$$

where $W(\boldsymbol{u})=\left(\boldsymbol{\nabla} \boldsymbol{u}-\boldsymbol{\nabla} \boldsymbol{u}^{T}\right) / 2$, and $a \in[-1,1]$. Note that Ozenda et al. (2018) used an upper-convected derivative, corresponding to $a=1$. Equation (2.1a) has no characteristic time, such that its solutions are rate-independent: In simple shear, changing the amplitude of the strain rate $\dot{\gamma}$ leaves the evolution of $\dot{\gamma}_{e}$ unchanged when expressed in terms of the dimensionless time $|\dot{\gamma}| t$ (that interprets as a strain).

The Cauchy stress tensor $\boldsymbol{\sigma}$ of the suspension is assumed to express as an analytical function of the two tensorial variables $\dot{\gamma}$ and $\gamma_{e}$. Following Hand (1962), and by virtue of Cayley-Hamilton theorem, only the zeroth, first and second powers of $\dot{\gamma}$ and $\gamma_{e}$ can contribute to the expression. Furthermore, to preserve rate-independence, terms in $\dot{\gamma}^{2}$ should also be discarded. After extensive tests to assess the effects of the different possible terms, we chose to consider the following expression for the Cauchy stress tensor:

$$
\boldsymbol{\sigma}=-p_{b} \boldsymbol{I}+\eta \dot{\gamma}+\eta_{e}\left\{\delta_{1}|\dot{\gamma}| \gamma_{e}+\beta\left(\frac{\gamma_{e}^{2} \dot{\gamma}+\dot{\gamma} \gamma_{e}^{2}}{2}-|\dot{\gamma}| \gamma_{e}^{2}\right)+\delta_{2}\left(\frac{\gamma_{e} \dot{\gamma}+\dot{\gamma} \gamma_{e}}{2}\right)\right\}
$$

where $p_{b}$ is the bulk pressure of the suspension, $\eta$ and $\eta_{e}$ are characteristic viscosities, and $\beta$ and $\delta_{2}$ are additional dimensionless material parameters. Note that the terms in factor of $\delta_{1}$ and $\delta_{2}$ are linear in $\gamma_{e}$, while the term in factor of $\beta$ is quadratic in $\gamma_{e}$.

The present model represents an upgrade of that presented by Ozenda et al. (2018), which involved only one linear and one non-linear terms. For the sake of simplicity and of formulating a minimal model, the scalar coefficients $\eta, \eta_{e} \beta, \eta_{e} \delta_{1}$ and $\eta_{e} \delta_{2}$ are assumed to be independent of the invariants of $\gamma_{e}$, which is a restriction compared to the general theory of Hand (1962).

Constitutive equations (2.1) are coupled with mass and momentum conservations to obtain a closed problem for three unknowns, namely the suspension pressure $p_{b}$, the suspension velocity $\boldsymbol{u}$, and the tensor describing the anisotropy of the micro-structure $\gamma_{e}$ : 


$$
\begin{array}{r}
\frac{\mathscr{D}_{a} \gamma_{e}}{\mathscr{D} t}+\delta_{1}|\dot{\gamma}| \gamma_{e}-\dot{\gamma}=0 \\
\rho\left(\frac{\partial \boldsymbol{u}}{\partial t}+(\boldsymbol{u} . \boldsymbol{\nabla}) \boldsymbol{u}\right)-\operatorname{div} \boldsymbol{\sigma}=0 \\
\operatorname{div} \boldsymbol{u}=0
\end{array}
$$

where the Cauchy stress tensor $\boldsymbol{\sigma}$ is expressed by $(2.1 c)$ and the strain rate tensor $\dot{\gamma}$ is expressed by $(2.1 b)$. Note that $p_{b}$ in $(2.1 c)$ should be regarded as a Lagrange multiplier associated to the incompressibility constraint $(2.2 c)$. This set of equations is closed by appropriate boundary and initial conditions for $\boldsymbol{u}$ and $\gamma_{e}$.

From $(2.2 a)$, remark that the conformation tensor $\boldsymbol{b}_{e}=c_{0}^{-1}\left(c_{0} \boldsymbol{I}+\boldsymbol{\gamma}_{e}\right)$ satisfies:

$$
\frac{\mathscr{D}_{a} \boldsymbol{b}_{e}}{\mathscr{D} t}-c_{0} \delta_{1}|\dot{\gamma}| \boldsymbol{I}+c_{0} \delta_{1}|\dot{\gamma}| \boldsymbol{b}_{e}=\left(1-a c_{0}\right) \dot{\gamma}
$$

Hence, by choosing the dimensionless constant $c_{0}=1 / a$, the right-hand-side in the previous relation is zero. In the following, we also assume $a \in] 0,1]$. According to Hulsen (1990), and since $\delta_{1}>0$, these choices guarantee that the conformation tensor $\boldsymbol{b}_{e}$ is positive definite at any time, if this property is satisfied at initial time.

\section{Simple shear and shear-reversal}

\subsection{The reduced problem}

Let us consider a simple shear flow with uniform shear rate. The $x$ axis is in the flow direction and the $y$ axis is in the direction of velocity gradient, such that $\boldsymbol{u}(t, x, y, z)=$ $\left(u_{x}(t, y), 0,0\right)$. Let us denote $\dot{\gamma}=\partial_{y} u_{x}$ the uniform scalar shear rate, such that $|\dot{\gamma}|=|\dot{\gamma}|$. Evolution equation $(2.2 a)$ for $\gamma_{e}(t)$ then reduces to the following system of ordinary differential equations:

$$
\begin{aligned}
& \partial_{t} \gamma_{e, x x}-(1+a) \dot{\gamma} \gamma_{e, x y}+\delta_{1}|\dot{\gamma}| \gamma_{e, x x}=0 \\
& \partial_{t} \gamma_{e, y y}+(1-a) \dot{\gamma} \gamma_{e, x y}+\delta_{1}|\dot{\gamma}| \gamma_{e, y y}=0 \\
& \partial_{t} \gamma_{e, x y}-\frac{1+a}{2} \dot{\gamma} \gamma_{e, y y}-\frac{1-a}{2} \dot{\gamma} \gamma_{e, x x}+\delta_{1}|\dot{\gamma}| \gamma_{e, x y}=\dot{\gamma} \\
& \partial_{t} \gamma_{e, x z}-(1+a) \dot{\gamma} \gamma_{e, y z}+\delta_{1}|\dot{\gamma}| \gamma_{e, x z}=0 \\
& \partial_{t} \gamma_{e, y z}+(1-a) \dot{\gamma} \gamma_{e, x z}+\delta_{1}|\dot{\gamma}| \gamma_{e, y z}=0 \\
& \partial_{t} \gamma_{e, z z}+\delta_{1}|\dot{\gamma}| \gamma_{e, z z}=0
\end{aligned}
$$


We assume $\gamma_{e, x z}=\gamma_{e, y z}=\gamma_{e, z z}=0$ at $t=0$, such that these components remain zero at any time. The constitutive equation $(2.1 c)$ for $\boldsymbol{\sigma}$ becomes:

$$
\begin{aligned}
& \sigma_{x x}=-p_{b}+\eta_{e}\left\{\delta_{1}|\dot{\gamma}| \gamma_{e, x x}+\beta\left(\dot{\gamma}\left(\gamma_{e, x x}+\gamma_{e, y y}\right) \gamma_{e, x y}-|\dot{\gamma}|\left(\gamma_{e, x x}^{2}+\gamma_{e, x y}^{2}\right)\right)+\delta_{2} \dot{\gamma} \gamma_{e, x y}\right\} \\
& \sigma_{y y}=-p_{b}+\eta_{e}\left\{\delta_{1}|\dot{\gamma}| \gamma_{e, y y}+\beta\left(\dot{\gamma}\left(\gamma_{e, x x}+\gamma_{e, y y}\right) \gamma_{e, x y}-|\dot{\gamma}|\left(\gamma_{e, x y}^{2}+\gamma_{e, y y}^{2}\right)\right)+\delta_{2} \dot{\gamma} \gamma_{e, x y}\right\} \\
& \sigma_{z z}=-p_{b} \\
& \sigma_{x y}=\eta \dot{\gamma}+\eta_{e}\left\{\delta_{1}|\dot{\gamma}| \gamma_{e, x y}+\beta\left(\frac{\dot{\gamma}}{2}\left(\gamma_{e, x x}^{2}+\gamma_{e, y y}^{2}+2 \gamma_{e, x y}^{2}\right)-|\dot{\gamma}|\left(\gamma_{e, x x}+\gamma_{e, y y}\right) \gamma_{e, x y}\right)\right. \\
& \left.+\frac{\delta_{2} \dot{\gamma}}{2}\left(\gamma_{e, x x}+\gamma_{e, y y}\right)\right\} \\
& \sigma_{x z}=\sigma_{y z}=0
\end{aligned}
$$

The system (3.1) is linear and admits explicit solutions when the shear rate $\dot{\gamma}$ is given. Explicit expressions of the stress components are then obtained from (3.2). In the following, we will also consider situations in which the shear stress $\sigma_{x y}$ is imposed. In this case, numerical solutions of the coupled equations (3.1)-(3.2) are computed using 1sode ordinary differential equation solver (Radhakrishnan \& Hindmarsh 1993) implemented within the numpy-scipy environment (Jones et al. 2001-).

\subsection{Explicit solution in steady-state}

The steady-state solution of $(3.1 a)-(3.1 c)$ writes:

$$
\begin{aligned}
\gamma_{e, x x} & =\frac{1+a}{1-a^{2}+\delta_{1}^{2}} \\
\gamma_{e, y y} & =\frac{-(1-a)}{1-a^{2}+\delta_{1}^{2}} \\
\gamma_{e, x y} & =\frac{\delta_{1} \operatorname{sgn}(\dot{\gamma})}{1-a^{2}+\delta_{1}^{2}}
\end{aligned}
$$

The steady-state apparent viscosity $\eta_{a p p}=\sigma_{x y} / \dot{\gamma}$ and normal stress differences $N_{1}=\sigma_{x x}-\sigma_{y y}$ and $N_{2}=\sigma_{y y}-\sigma_{z z}$ are then easily deduced from $(3.2 a)-(3.2 d)$ :

$$
\begin{aligned}
\eta_{a p p} & =\eta+\frac{\eta_{e}}{1-a^{2}+\delta_{1}^{2}}\left(\delta_{1}^{2}+a \delta_{2}+\frac{\left(\left(1+a^{2}+\delta_{1}^{2}\right)-2 a \delta_{1}\right) \beta}{1-a^{2}+\delta_{1}^{2}}\right) \\
N_{1} & =\frac{2 \eta_{e}|\dot{\gamma}|}{1-a^{2}+\delta_{1}^{2}}\left(\delta_{1}-\frac{2 a \beta}{1-a^{2}+\delta_{1}^{2}}\right) \\
N_{2} & =\frac{\eta_{e}|\dot{\gamma}|}{1-a^{2}+\delta_{1}^{2}}\left(\delta_{1}\left(-1+a+\delta_{2}\right)+\frac{\left(2 a \delta_{1}-\left((1-a)^{2}+\delta_{1}^{2}\right)\right) \beta}{1-a^{2}+\delta_{1}^{2}}\right)
\end{aligned}
$$

We also define the particle pressure $p_{p}=p-p_{b}$, where $p=-\operatorname{tr}(\boldsymbol{\sigma}) / 3$ is the total pressure of the suspension. In steady-state:

$$
p_{p}=-\frac{2 \eta_{e}|\dot{\gamma}|}{3\left(1-a^{2}+\delta_{1}^{2}\right)}\left(\delta_{1}\left(a+\delta_{2}\right)+\frac{\left(2 a \delta_{1}-\left(1+a^{2}+\delta_{1}^{2}\right)\right) \beta}{1-a^{2}+\delta_{1}^{2}}\right)
$$




\subsection{Dependence of material parameters upon volume fraction}

The rheological model presented in section 2 involves six material parameters: two viscosities $\eta$ and $\eta_{e}$, and four dimensionless parameters $a, \delta_{1}, \delta_{2}$ and $\beta$. Preliminary tests to fit experimental data for different volume fractions $\phi$ (see $\S 4$ ), showed that all these parameters, except $a$, have to vary with $\phi$. Following these preliminary investigations, we assumed $a$ to be independent of $\phi$, and the five remaining parameters to depend only upon the reduced volume fraction $\psi=\phi / \phi_{m}$, with $\phi_{m}$ the maximal volume fraction of the suspension. This latter assumption is required to obtain a model that can be applied to different experimental datasets characterised by different values of $\phi_{m}$. In practice, $\phi_{m}$ can vary between 0.53 and 0.64 , typically, depending on particle shape, particle roughness, etc (e.g., Guazzelli \& Pouliquen 2018).

As shown in appendix A, the parameter $\delta_{1}$ is directly related to the depletion angle $\theta_{e}$, i.e. the angle between the $x$ axis and the direction of the eigenvector associated to the largest eigenvalue of $\gamma_{e}: \delta_{1}=\tan \left(2 \theta_{e}\right)$. Based on the experimental measurements of $\theta_{e}$ provided by Blanc et al. (2013), the following dependence law is proposed for $\delta_{1}$ (see figure 1):

$$
\delta_{1}(\psi)=\bar{\delta}_{1}\left((1-\psi)^{-1}-(1-b \psi)\right),
$$

where $\bar{\delta}_{1}$ and $b$ are positive constants independent of $\psi$. Observe that $\delta_{1}(0)=0$ and $\delta_{1}(1)=\infty$, such that the model predicts $\theta_{e}(0)=0$ and $\theta_{e}(1)=\pi / 4$.

To be consistent with numerous existing results (Maron \& Pierce 1956; Morris \& Boulay 1999; Guazzelli \& Pouliquen 2018), the apparent viscosity $\eta_{a p p}$ and the second normal stress difference $N_{2}$ should behave as $(1-\psi)^{-2}$ in the $\psi \rightarrow 1$ limit. In addition, we expect $\eta_{e}=0$ in the Newtonian limit $\psi=0$. Accordingly, the following dependence laws are postulated for the viscosities $\eta$ and $\eta_{e}$ :

$$
\begin{aligned}
\eta(\psi) & =\eta_{0}\left(1-\omega+\psi\left(\frac{5}{2} \phi_{m}-2 \omega\right)+\omega(1-\psi)^{-2}\right) \\
\eta_{e}(\psi) & =\eta_{0} \bar{\eta}_{e} \psi(1-\psi)^{-2}
\end{aligned}
$$

where $\eta_{0}$ is the viscosity of the suspending fluid, and $\bar{\eta}_{e}>0$ is a constant independent of $\psi$. The constant $\omega \in] 0,1$ [ is introduced in (3.5b) in order to recover Einstein (1906)'s relation when $\psi \rightarrow 0$ (see $\S 3.4$ ). Note that $\eta$ expresses as a convex combination of Maron \& Pierce (1956) and Einstein (1906) laws.

Similar to $\eta_{e}$, the parameters $\delta_{2}$ and $\beta$ should vanish when $\psi=0$, i.e. when the suspension reduces to a Newtonian fluid. For simplicity, we consider that $\delta_{2}$ is proportional to $\delta_{1}$. We also assume that $\beta$ behave as $(1-\psi)^{-2}$ when $\psi \rightarrow 1$. Accordingly, the following dependence laws are postulated:

$$
\begin{aligned}
\delta_{2}(\psi) & =\bar{\delta}_{2}\left((1-\psi)^{-1}-(1-b \psi)\right) \\
\beta(\psi) & =\bar{\beta}\left((1-\psi)^{-1}-1\right)^{2}, \quad i=1,2,3
\end{aligned}
$$

where $\bar{\delta}_{2}$ and $\bar{\beta}$ are constants independent of $\psi$.

At this stage, the suspension model involves seven constants independent of $\psi$, namely $a, b, \omega, \bar{\eta}_{e}, \bar{\delta}_{1}, \bar{\delta}_{2}$, and $\bar{\beta}$ that need to be determined from experimental data. Note that with the dependence laws $(3.5 b)-(3.5 c)$ for $\eta$ and $\eta_{e}$, we obtain from (3.4) that the normal stress ratios

$$
\alpha_{1}=\frac{N_{1}}{\eta_{a p p}|\dot{\gamma}|} \quad \text { and } \quad \alpha_{2}=\frac{N_{2}}{\eta_{a p p}|\dot{\gamma}|}
$$


are independent of suspending fluid viscosity $\eta_{0}$, in agreement with experiments (Dbouk et al. 2013; Guazzelli \& Pouliquen 2018).

\subsection{Dilute and concentrated limits}

In the dilute limit $\psi \rightarrow 0$, a second-order Taylor expansion of (3.4a) leads to the following expression for apparent viscosity $\eta_{a p p}$ :

$$
\eta_{a p p}=\eta_{0}\left(1+\frac{5}{2} \phi_{m} \psi+\left(\frac{3 \omega}{2}+\frac{a(1+b) \bar{\delta}_{2} \bar{\eta}_{e}}{\left(1-a^{2}\right)}\right) \psi^{2}\right)+O\left(\psi^{3}\right)
$$

At first order in $\psi$, the model agrees with Einstein (1906)'s relation $\eta_{a p p} \approx \eta_{0}(1+5 \phi / 2)$. The different constants associated to micro-structure evolution, $a, b, \bar{\eta}_{e}$ and $\bar{\delta}_{2}$, appear only at second order, consistently with the asymptotic expansion of Batchelor \& Green (1972).

In the concentrated limit $\psi \rightarrow 1$, the expansion of $\eta_{a p p}$ writes:

$$
\begin{aligned}
\eta_{\text {app }} & =\eta_{0} \bar{\eta}_{1}(1-\psi)^{-2}+O\left((1-\psi)^{-1}\right) \\
\text { with } \bar{\eta}_{1} & =\left(\omega+\left(1+\frac{\bar{\beta}}{\bar{\delta}_{1}^{2}}\right) \bar{\eta}_{e}\right)
\end{aligned}
$$

Hence, $\eta_{a p p}$ grows as $(1-\psi)^{-2}$, as required (Maron \& Pierce 1956).

Let us now turn to the asymptotic behaviour of normal stress ratios $\alpha_{1}$ and $\alpha_{2}$ and particle pressure $p_{p}$. In the dilute limit $\psi \rightarrow 0$, from (3.4), we obtain:

$$
\begin{aligned}
\alpha_{1} & =\frac{2 \bar{\eta}_{e} \bar{\delta}_{1}(1+b)}{1-a^{2}} \psi^{2}+O\left(\psi^{3}\right) \\
\alpha_{2} & =-\frac{(1-a) \bar{\eta}_{e} \bar{\delta}_{1}(1+b)}{1-a^{2}} \psi^{2}+O\left(\psi^{3}\right) \\
\frac{p_{p}}{\eta_{a p p}|\dot{\gamma}|} & =-\frac{2 a \bar{\eta}_{e} \bar{\delta}_{1}(1+b)}{3\left(1-a^{2}\right)} \psi^{2}+O\left(\psi^{3}\right)
\end{aligned}
$$

Observe that $\alpha_{1}, \alpha_{2}$ and $p_{p}$ all vanish for $\psi=0$, as expected. Moreover, we obtain $\alpha_{1} \geqslant 0$ and $\alpha_{2} \leqslant 0$ when $\psi \rightarrow 0$. The sign of $\alpha_{2}$ is consistent with experimental observations (Couturier et al. 2011; Dbouk et al. 2013; Dai et al. 2013).

In the concentrated limit $\psi \rightarrow 1$, the expansions of normal stress ratios and particle pressure write:

$$
\begin{aligned}
\alpha_{1} & =\frac{2 \bar{\eta}_{e}}{\bar{\eta}_{1} \bar{\delta}_{1}}(1-\psi)+O\left((1-\psi)^{2}\right) \\
\alpha_{2} & =-\frac{\bar{\eta}_{e}}{\bar{\eta}_{1}}\left(\frac{-\bar{\delta}_{1} \bar{\delta}_{2}+\bar{\beta}}{\bar{\delta}_{1}^{2}}\right)+O(1-\psi) \\
\frac{p_{p}}{\eta_{a p p}|\dot{\gamma}|} & =\frac{2 \bar{\eta}_{e}}{3 \bar{\eta}_{1}}\left(\frac{-\bar{\delta}_{1} \bar{\delta}_{2}+\bar{\beta}}{\bar{\delta}_{1}^{2}}\right)+O(1-\psi)
\end{aligned}
$$

Hence, the ratio $\alpha_{1}$ vanishes when $\psi \rightarrow 1$. On the contrary, $\alpha_{2}$ tends to a constant for $\psi \rightarrow 1$, in agreement with experiments (Leighton \& Acrivos 1987; Guazzelli \& Pouliquen 2018). Furthermore, it is sufficient to assume $\bar{\delta}_{2}<0$ and $\bar{\beta} \geqslant 0$ to obtain $\alpha_{2} \leqslant 0$. 


\begin{tabular}{ccccccc}
\hline$a$ & $b$ & $\omega$ & $\bar{\eta}_{e}$ & $\bar{\delta}_{1}$ & $\bar{\delta}_{2}$ & $\bar{\beta}$ \\
\hline 0.51 & 1.5 & 0.52 & 0.089 & 0.47 & -1.15 & 1.7 \\
\hline
\end{tabular}

TABLE 1. Values of the seven dimensionless model constants identified from experimental data.

\section{Quantitative comparisons with experiments}

\subsection{Identification of model constants}

As explained above, the rheological model proposed in this paper was built to recover qualitative agreement with experimental observations in the dilute and concentrated limits. More quantitatively, the seven model constants were identified through an adjustment to different experimental datasets reporting detailed rheological measurements on non-colloidal suspensions for different volume fractions $\phi$ (in the range $[0.2,0.5])$. The four following datasets were considered for the adjustment:

- Measurements of the depletion angle $\theta_{e}$ performed by Blanc et al. (2013). These authors used a suspending fluid of viscosity $\eta_{0}=0.85$ Pa.s and two sets of PMMA spheres of radius $r_{p}=80$ and $90 \mu \mathrm{m}$. They reported a maximal volume fraction $\phi_{m}=0.57$.

- Measurements of transient apparent viscosity after a shear-reversal performed by Blanc et al. (2011) for five different values of volume fraction $\phi$. These authors used a suspending fluid of viscosity $\eta_{0}=1.02 \mathrm{~Pa} . \mathrm{s}$, PMMA spheres of radius $r_{p}=16 \mu \mathrm{m}$, and reported a relatively low maximal volume fraction $\phi_{m}=0.535$ presumably due to the presence of a residual cellulosic surfactant.

- Measurements of normal-stress differences in steady-state performed by Couturier et al. (2011). These authors used a suspending fluid with viscosity $\eta_{0}=2.15$ Pa.s, polystyrene spheres of radius $r_{p}=35 \mu \mathrm{m}$, and reported a maximal volume fraction $\phi_{m}=0.62$.

- Measurements of normal-stress differences in steady-state performed by Dai et al. (2013). These authors used a slightly shear-thinning suspending fluid with viscosity $1.17 \leqslant \eta_{0} \leqslant 1.3$ Pa.s for shear rates $0.01 \leqslant \dot{\gamma} \leqslant 100 \mathrm{~s}^{-1}$, polystyrene spheres of radius $r_{p}=20 \mu \mathrm{m}$, and reported a maximal volume fraction $\phi_{m}=0.62$.

For all these datasets, values of $\phi$ were rescaled in terms of reduced volume fraction $\psi=\phi / \phi_{m}$ by using the provided values of $\phi_{m}$. As described in appendix $\mathrm{B}$, the set of seven model constants $\left\{a, \omega, \bar{\eta}_{e}, \bar{\delta}_{2}, \bar{\beta}_{1}, \bar{\beta}_{2} \bar{\beta}_{3}\right\}$ was globally identified from the datasets. The obtained values are indicated in table 1 . Note that first attempts at trying to obtain a comparable quantitative agreement with both steady-state and transient measurements, failed. As a consequence, we chose to assign a stronger weight to the agreement with steady-state viscosity and normal-stress data. A lower weight was assigned to the agreement with transient experiments, although sufficient to ensure that model predictions present a correct qualitative behavior. More details on the identification procedure, including expression of the minimised cost function, are provided in appendix B.

Direct comparisons between model predictions with the constants given in table 1, and the experimental data used for the identification, are shown in figures 1,2 and 3 . 

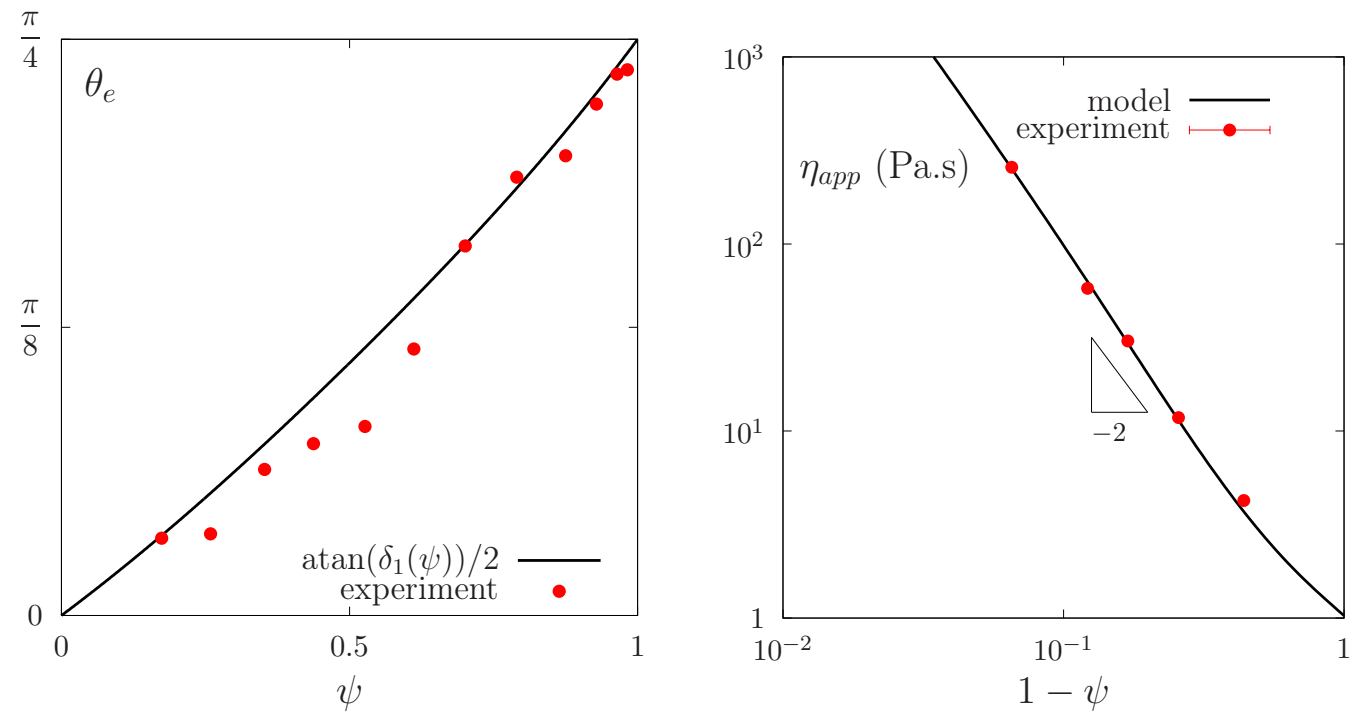

FiguRE 1. Evolution of depletion angle $\theta_{e}$ and steady-state apparent viscosity $\eta_{a p p}$ versus reduced volume fraction $\psi$ : comparison between model predictions and experimental data of Blanc et al. (2013) $\left(\theta_{e}\right)$ and Blanc et al. (2011) $\left(\eta_{a p p}\right)$.
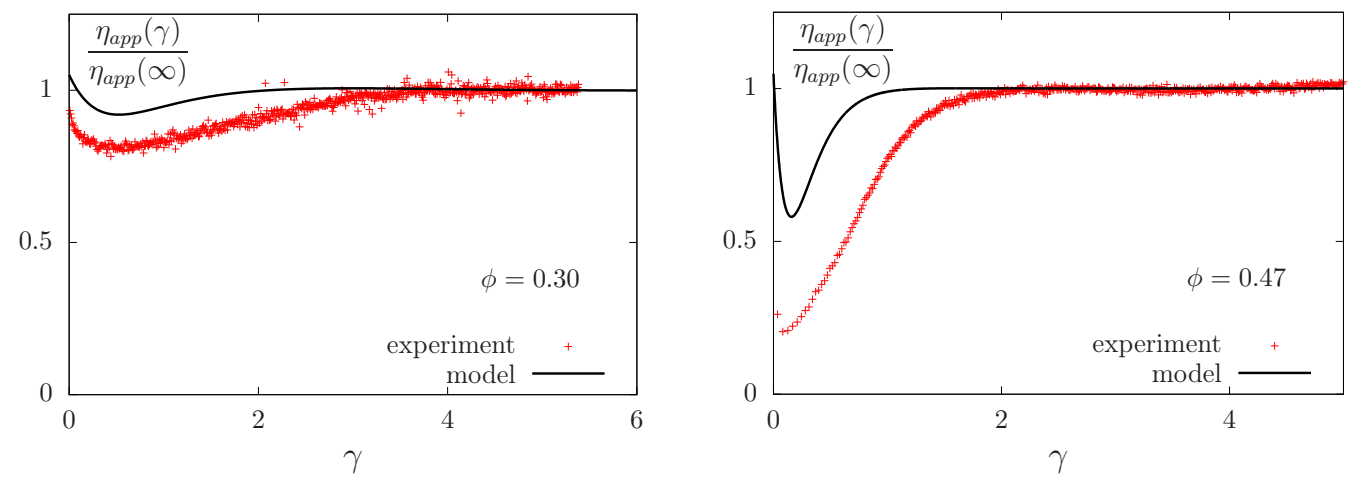

FiguRE 2. Evolution of apparent viscosity $\eta_{a p p}$ versus shear strain $\gamma$ after shear-reversal: comparison between model prediction and experimental measurements of Blanc et al. (2011) for two volume fractions $\phi$.

Figure 1 shows that predicted steady-state depletion angle and apparent viscosity are indeed in excellent quantitative agreement with the experimental data. Figure 2 presents comparisons for the transient response, namely evolution of apparent viscosity $\eta_{a p p}(t)$ versus strain $\gamma(t)=\int_{0}^{t}|\dot{\gamma}(s)| \mathrm{d} s$ after a shear-reversal. Let us recall that the experiments of Blanc et al. (2011) were performed at imposed shear stress, while $\dot{\gamma}(t)$ varies. Note that both model predictions and experimental data are normalised by their respective steady state-values $\eta_{a p p}(\infty)$ in the figure. It is observed that, although the model generally tends to underestimate the amplitude of the transient response, a satisfactory qualitative behavior is nevertheless obtained for all values of $\phi$. In particular, the successive phases present in the measurements, namely the initial brutal drop in apparent viscosity, the smooth evolution to a minimum, and the relaxation towards the steady-state, are all reproduced. The typical duration of the transient phase is also correctly captured.

Figure 3 presents the evolution of steady-state normal stress ratios $\alpha_{1}$ and $\alpha_{2}$ as a 

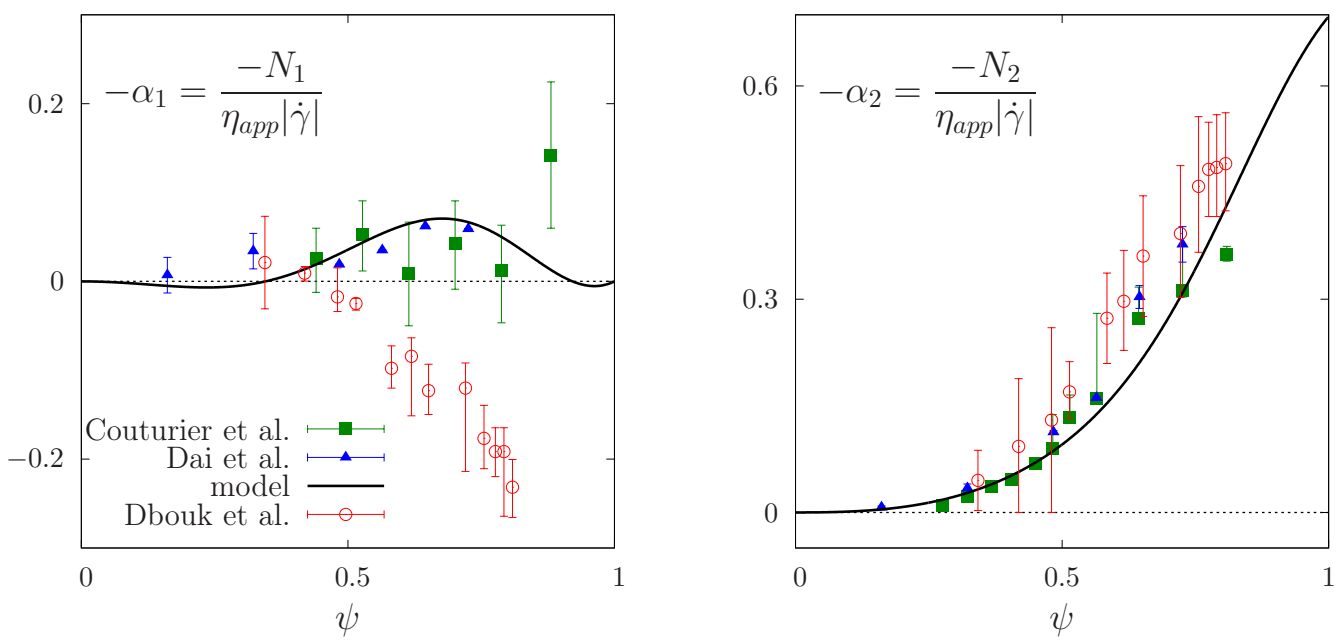

FiguRE 3. Evolution of steady-state normal stress ratios $\alpha_{1}$ and $\alpha_{2}$ versus reduced volume fraction $\psi$ : comparison between model predictions and experimental measurements of Couturier et al. (2011), Dai et al. (2013) and Dbouk et al. (2013).

function of reduced volume fraction $\psi$. The model appears to successfully capture the main features of the normal stress measurements reported in the studies of Couturier et al. (2011) and Dai et al. (2013), namely $N_{2}<0$ and $\left|N_{1}\right|<\left|N_{2}\right|$. Predictions for the second normal-stress ratio $\alpha_{2}$ show a strong monotonic decrease with $\psi$, and are in excellent quantitative agreement with the data (figure 3-right). Regarding the first normal-stress difference, the model predicts non-monotonic variations of the ratio $\alpha_{1}$ with $\psi$ (figure 3-left). Values of $\alpha_{1}$ are positive but very small for $\psi \lesssim 0.4$, negative and decreasing with $\psi$ for $0.4 \lesssim \psi \lesssim 0.7$, and then increasing again towards the concentrated limit $\alpha_{1}(1)=0$ (see $\S 3.4$ ). Here also, these predictions appear to be in good quantitative agreement with the measurements in terms of overall magnitude, albeit the existence of non-monotonic variations cannot be confirmed from existing data due to the level of experimental noise. For comparison, experimental measurements obtained by Dbouk et al. (2013) (not used for the adjustment) are also shown in figure 3, using a value $\phi_{m}=0.58$ for the volume fraction scaling. These measurements are fully consistent with the two other datasets and the model for $\alpha_{2}$, but show positive values of $\alpha_{1}$, thereby highlighting the need for additional characterisations of the first normal-stress difference in these systems.

\subsection{Comparison with independent particle pressure measurements}

Model predictions were also assessed against steady-state particle pressure measurements obtained by Deboeuf et al. (2009). These authors used a suspending fluid of viscosity $\eta_{0}=3 \mathrm{~Pa} . \mathrm{s}$ and differents sets of particles with radii ranging between 40 and $140 \mu \mathrm{m}$. As they did not provide the value of maximum volume fraction $\phi_{m}$ in their systems, we used $\phi_{m}=0.58$ to rescale their data. We insist that this new dataset was not used for the identification of model constants. As shown in figure 4, model predictions obtained with the values of constants given in table 1, show here also a good agreement with the experimental measurements. This remarkable result validates the introduction of reduced volume fraction $\psi=\phi / \phi_{m}$ in the dependence laws for the material parameters (see §3.3), and indicates that the model constants involved in these dependence laws can be regarded, at least to a first approximation, as independent of the experimental 


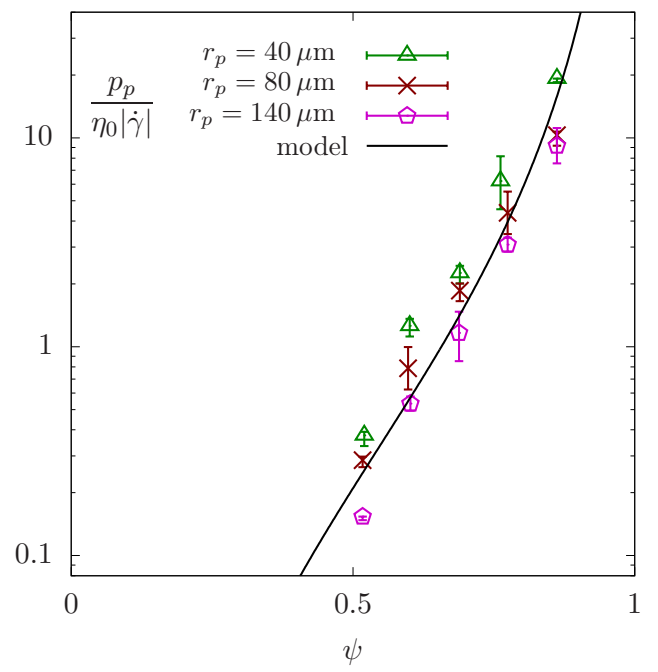

FiguRE 4. Evolution of steady-state normalized particle pressure $p_{p} /\left(\eta_{0}|\dot{\gamma}|\right)$ versus reduced volume fraction $\psi$ : comparison between model predictions and experimental measurements of Deboeuf et al. (2009). The consistent data obtained for three different values of particle radius $r_{p}$ are shown.

conditions. In other words, the influence of experimental conditions is fully encoded in the maximum volume fraction $\phi_{m}$ used to rescale the volume fraction values.

\section{Sensitivity study}

A systematic sensitivity study of model predictions with respect to the seven model constants $a, b, \omega, \bar{\eta}_{e}, \bar{\beta}, \bar{\delta}_{1}, \bar{\delta}_{2}$, was performed. Figure 5 shows the evolution of steadystate normal stress ratios $\alpha_{1}$ and $\alpha_{2}$ and steady-state apparent viscosity $\eta_{a p p}$ versus $\psi$ (upper three plot lines), as well as the transient evolution of $\eta_{a p p}$ after a shear-reversal (lower plot line), when model constants are varied in consistent $[-50 \%,+100 \%]$ ranges around their reference values obtained through identification (see $\S 4$ ).

It is observed that a variation of any of the model constant implies a significant effect on at least one of the displayed quantities. Hence, none of the terms introduced in the Cauchy stress equation $(2.1 c)$ can be omitted without significantly degrading the match with experimental observations. We also note the existence of non-trivial couplings between these different terms, which explains the difficulties encountered in the identification procedure when trying to simultaneously reproduce steady-state and transient experimental data (see $\S 4$ ). Note that unphysical predictions $\left|\alpha_{1}\right| \geqslant\left|\alpha_{2}\right|$ are obtained when values of $\bar{\delta}_{1}$ are close to zero, values of $\bar{\beta}$ are too high, or when $a \approx 1$.

A closer look on the results provides insights into the physical interpretation of the different model constants. In steady-state, the constant $\bar{\eta}_{e}$ acts as a magnitude scaling for all stress terms that are non-colinear to the strain rate $\dot{\gamma}$ (see (3.4)). Accordingly, this affects both normal stress ratios $\alpha_{1}$ and $\alpha_{2}$. Conversely, $\bar{\beta}$ only acts on terms that are quadratic in $\dot{\gamma}$, and mostly affects the first normal stress ratio $\alpha_{1}$. Both $\bar{\eta}_{e}$ and $\bar{\beta}$ also play a role on the steady-state viscosity $\eta_{a p p}$, but have essentially no influence on the minimum observed during the transient evolution of $\eta_{a p p}$.

The constant $\bar{\delta}_{1}$ interprets as a characteristic strain controlling the length of the transients. In particular, this constant sets the position of the minimum in $\eta_{a p p}$. This 


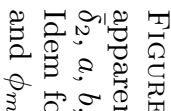

इ क्षे

نे

प्य

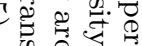

ब.

过它 8

龸蛋. 蛋

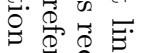

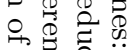

है ठ है

ڤ્仓 ठీ

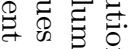

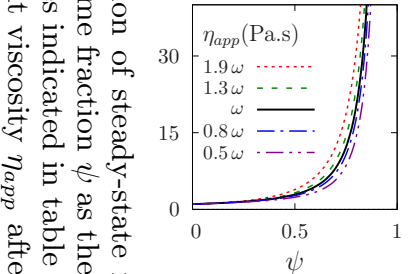

$\rightarrow \vdash \stackrel{0}{\infty}$

थ 2 की

कृ 0

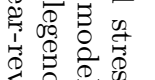

कृ क

की 8

की

Q ठ

II

i
Steady-state
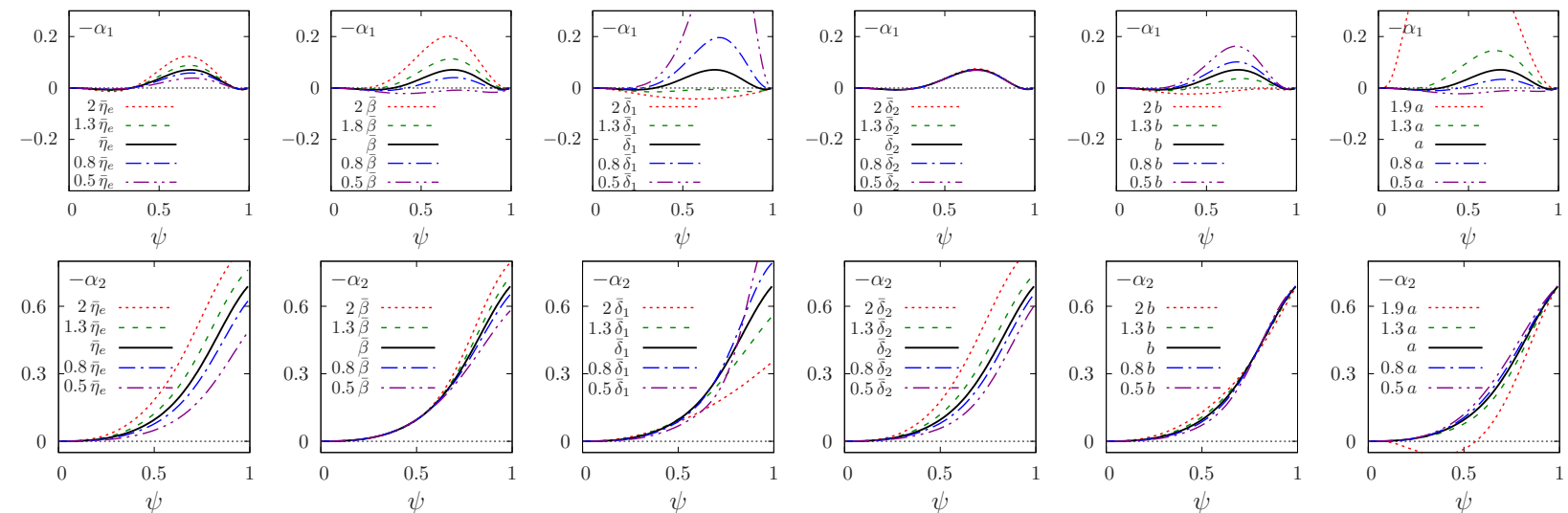

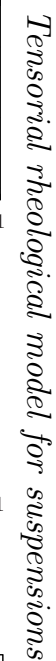

Shear-reversal
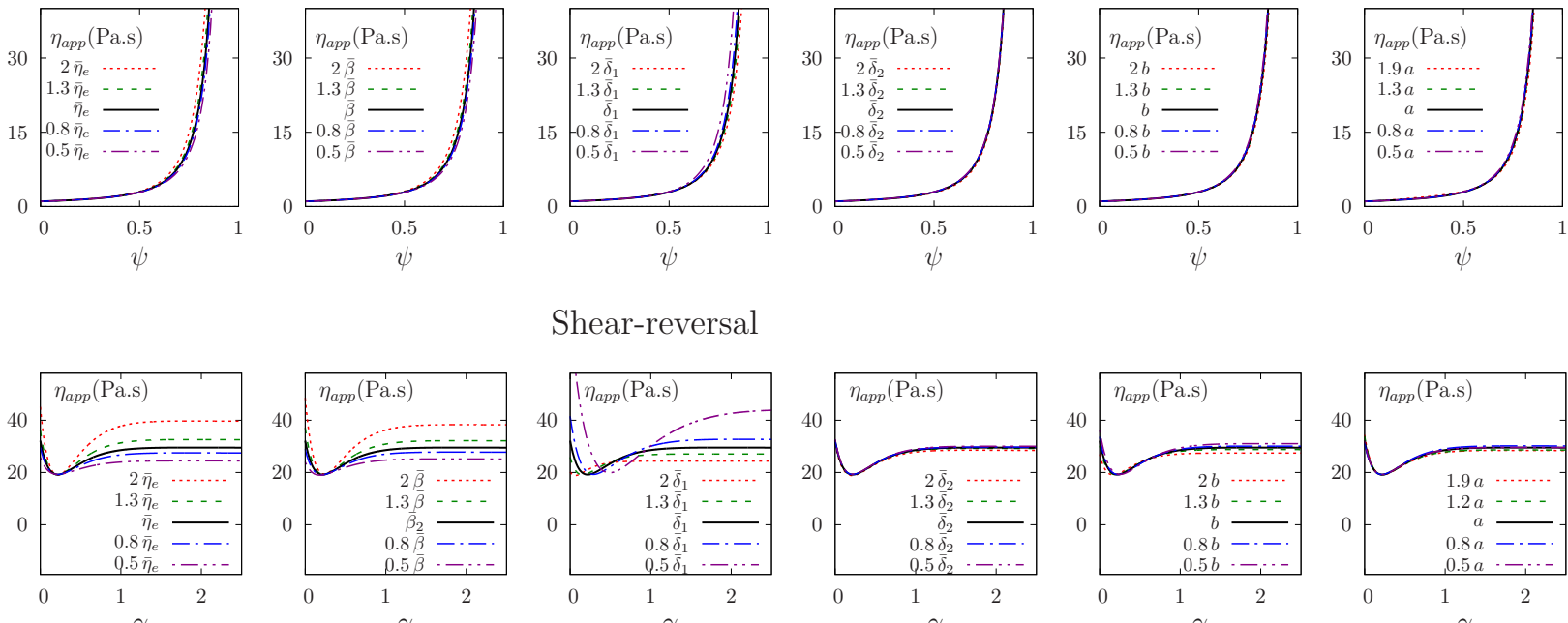
constant also plays a strong role on the steady-state values of apparent viscosity and normal-stress ratios. This ubiquitous role is consistent with the fact that $\delta_{1}$ is the only parameter present in both the constitutive equation for $\boldsymbol{\sigma}(2.1 c)$ and the evolution equation for $\gamma_{e}(2.2 a)$.

The constants $\bar{\delta}_{2}, a$ and $b$ do not have much influence on the apparent viscosity $\eta_{a p p}$. The constant $\bar{\delta}_{2}$ essentially acts on the second normal stress ratio $\alpha_{2}$, while the constants $a$ and $b$ have a more pronounced effect on $\alpha_{1}$. However, an unphysical change of sign of $\alpha_{2}$ is observed when $a \approx 1$. These observations are consistent with equations (3.4).

Finally, the constant $\omega$ is responsible for an overall shift of the apparent viscosity $\eta_{a p p}$ in both transient and steady-state regimes, but only weakly affects the normal stress ratios $\alpha_{1}$ and $\alpha_{2}$.

\section{Discussion and conclusions}

An improved, rate-independent rheological model for non-colloidal suspensions of rigid spheres, involving the evolution of a conformation tensor, was presented. The model upgrades that of Ozenda et al. (2018) in several aspects. While the structure of the linear evolution equation (2.1a) for the conformation tensor is unchanged, a more general Gordon-Schowalter tensor derivative is used, with a material parameter $a \in] 0,1[$. In addition, the expression of the Cauchy stress tensor $(2.1 c)$ is modified by the inclusion of additional linear and non-linear terms. While still keeping with the goal of a minimal model involving as few adjustable parameters as possible, these amendments were required to predict both the transient evolution of apparent viscosity after a shearreversal, and realistic values for the normal stress difference $N_{1}$ and $N_{2}$ in steady-state. Although the contributions of the different terms to the overall rheological behavior of the suspension appear to be strongly coupled, a systematic sensitivity study was performed to provide insights into the physical interpretation of the different model parameters. Note however that the sensitivity study was performed for limited ranges of the parameters, and might thus not be representative of the model behavior when parameters are varied in much larger ranges.

The model was compared to different experimental datasets involving various suspending fluids and particle sizes. To that aim, the material parameters were all expressed in terms of reduced volume fraction $\psi=\phi / \phi_{m}$, where the maximum volume fraction $\phi_{m}$ varies among the experiments, and a of set of seven model constants. The good agreement observed with steady-state data, in terms of apparent viscosity, normal stress differences and particle pressure, suggests that the rheological model, together with the constants given in table 1, can be applied to large range of non-colloidal suspensions. Regarding the transient responses, only qualitative agreement was obtained with experimental data. Further improvements of these transient predictions would certainly require complementing our minimal model with additional terms, for instance polynomial dependences of the material parameters upon the invariants of the texture tensor (Hand 1962), or introduction of additional microstructural tensorial variables (Goddard 2006). Along the same line, introduction of a plasticity term in the constitutive law would open interesting perspectives to tackle highly-concentrated regimes close to, or above, the jamming transition, in which the rheological behavior becomes dominated by sustained granular contacts (Pailha \& Pouliquen 2009; Baumgarten \& Kamrin 2019). 
The capability of the presented model to predict accurate normal-stress differences in steady-state potentially opens way to computations of elongational or more complex flows. Such applications to a larger class of flows, together with a more systematic parameter identification procedure, shall also make it possible to improve the physical interpretation of the different terms introduced in the expression of the Cauchy stress tensor. Here also, detailed comparisons with experiments (e.g. Dai \& Tanner 2017) will however be necessary to assess the validity of the model, and particularly of the dependences of material parameters upon volume fraction introduced in $\S 3.3$, beyond the simple shear configuration investigated in this study. A more in-depth analysis of the mathematical and thermodynamical properties of the model would also need to be undertaken. The new rheological model could also be coupled to an evolution equation for the volume fraction in order to address particle migration processes (e.g., Miller \& Morris 2006; Haddadi et al. 2014), test the effect of normal-stress differences over longer time scales, and allow for comparisons with other modelling approaches (e.g., Dbouk 2016). Finally, let us also note that, although the focus of the present paper was mainly on trying to reproduce physical experiments, systematic comparisons with discrete simulation results (e.g. Chacko et al. 2018; Singh et al. 2018) could constitute a fruitful avenue for further validating and improving our rheological model. In particular, comparisons regarding the evolution of normal stress differences during transients, for which no experimental data are currently available, could provide useful additional insights.

\section{Declaration of interests}

The authors report no conflict of interest.

\section{REFERENCES}

Batchelor, G. K. \& Green, J. T. 1972 The determination of the bulk stress in a suspension of spherical particles to order $c^{2}$. J. Fluid Mech. 56 (03), 401-427.

Baumgarten, A. S. \& Kamrin, K. 2019 A general fluid-sediment mixture model and constitutive theory validated in many flow regimes. J. Fluid Mech. 861, 721-764.

Blanc, F., Lemaire, E., Meunier, A. \& Peters, F. 2013 Microstructure in sheared nonbrownian concentrated suspensions. J. Rheol. 57 (1), 273-292.

Blanc, F., Peters, F. \& Lemaire, E. 2011 Local transient rheological behavior of concentrated suspensions. J. Rheol. 55 (4), 835-854.

Boyer, F., Pouliquen, O. \& Guazzelli, E 2011 Dense suspensions in rotating-rod flows: normal stresses and particle migration. J. Fluid Mech. 686, 5-25.

Brady, John F \& Bossis, Georges 1985 The rheology of concentrated suspensions of spheres in simple shear flow by numerical simulation. J. Fluid Mech. 155, 105-129.

Chacko, R. N., Mari, R., Fielding, S. M \& Cates, M. E. 2018 Shear reversal in dense suspensions: the challenge to fabric evolution models from simulation data. J. Fluid Mech. 847, 700-734.

Couturier, E., Boyer, F., Pouliquen, O. \& Guazzelli, E 2011 Suspensions in a tilted trough: second normal stress difference. J. Fluid Mech. 686, 26-39.

DAi, S. \& TAnner, R. I. 2017 Elongational flows of some non-colloidal suspensions. Rheol. Acta $56(1), 63-71$.

Dai, S.-C., Bertevas, E., QI, F. \& Tanner, R. I. 2013 Viscometric functions for noncolloidal sphere suspensions with newtonian matrices. J. Rheol. 57 (2), 493-510.

Dвоuk, T. 2016 A suspension balance direct-forcing immersed boundary model for wet granular flows over obstacles. J. Non-Newt. Fluid Mech. 230, 68-79. 
Dbouk, T., Lobry, L. \& Lemaire, E. 2013 Normal stresses in concentrated non-brownian suspensions. J. Fluid Mech. 715, 239-272.

Deboeuf, A., Gauthier, G., Martin, J., Yurkovetsky, Y. \& Morris, J. F. 2009 Particle pressure in a sheared suspension: a bridge from osmosis to granular dilatancy. Phys. Rev. Let. 102 (10), 108301.

Denn, M. M. \& Morris, J. F. 2014 Rheology of non-brownian suspensions. Ann. Rev. Chem. and Biom. Eng. 5, 203-228.

Einstein, A. 1906 Eine neue bestimmung der moleküldimensionen. Ann. Phys. ser. 4 19, 289306.

Gadala-Maria, F 1979 The rheology of concentrated suspensions. PhD thesis, Stanford University.

Gadala-Maria, F. \& ACRivos, A. 1980 Shear-induced structure in a concentrated suspension of solid spheres. J. Rheol. 24 (6), 799-814.

Gallier, S., Lemaire, E., Peters, F. \& Lobry, L. 2014 Rheology of sheared suspensions of rough frictional particles. J. of Fluid Mech. 757, 514-549.

GodDARD, J. D. 1982 Memory materials without characteristic time and their relation to the rheology of certain particle suspensions. Adv. Coll. Interf. Sci. 17 (1), 241-262.

Goddard, J. D. 2006 A dissipative anisotropic fluid model for non-colloidal particle dispersions. J. Fluid Mech. 568, 1-17.

Gordon, R. J. \& Schowalter, W. R. 1972 Anisotropic fluid theory: a different approach to the dumbbell theory of dilute polymer solutions. J. Rheol. 16, 79-97.

Guazzelli, E. \& Pouliquen, O. 2018 Rheology of dense granular suspensions. J. Fluid Mech. 852, P1.

Haddadi, H., Shojaei-Zadeh, S., Connington, K. \& Morris, J. F. 2014 Suspension flow past a cylinder: particle interactions with recirculating wakes. J. Fluid Mech. 760, R2-1$\mathrm{R} 2-12$.

HAND, G. L. 1962 A theory of anisotropic fluids. J. Fluid Mech. 13 (1), 33-46.

Hulsen, M. A. 1990 A sufficient condition for a positive definite configuration tensor in differential models. J. Non-Newt. Flui Mech. 38 (1), 93-100.

JACKSON, R. 2000 The dynamics of fluidized particles. Cambridge university press.

Jones, E., Oliphant, T., Peterson, P. \& Others 2001- SciPy: Open source scientific tools for Python. [Online].

Kolli, V. G., Pollauf, E. J. \& Gadala-Maria, F. 2002 Transient normal stress response in a concentrated suspension of spherical particles. J. Rheol 46 (1), 321-334.

Lehoucq, R., Weiss, J., Dubrulle, B., Amon, A., le Boull, A., Crassous, J., Amitrano, D. \& Graner, F. 2015 Analysis of image vs. position, scale and direction reveals pattern texture anisotropy. Front. in Phys. 2, 84.

Leighton, D. \& ACrivos, A. 1987 The shear-induced migration of particles in concentrated suspensions. J. Fluid Mech. 181, 415-439.

Mari, R., Seto, R., Morris, J. F. \& Denn, M. M. 2014 Shear thickening, frictionless and frictional rheologies in non-brownian suspensions. J. Rheol. 58 (6), 1693-1724.

Maron, S. H. \& Pierce, P. E. 1956 Application of Ree-Eyring generalized flow theory to suspensions of spherical particles. J. Colloid Sci. 11 (1), 80-95.

Miller, R. M. \& Morris, J. F. 2006 Normal stress-driven migration and axial development in pressure-driven flow of concentrated suspensions. J. Non-Newt. Fluid Mech. 135 (2-3), 149-165.

More, J. J., Garbow, B. S. \& Hillstrom, K. E. 1980 User guide for minpack-1.[in fortran]. Tech. Rep..

Morris, J. F. \& Boulay, F. 1999 Curvilinear flows of noncolloidal suspensions: The role of normal stresses. J. Rheol. 43 (5), 1213-1237.

Newville, M., Stensitzki, T., Allen, D. B., Rawlik, M., Ingargiola, A. \& Nelson, A. 2016 Lmfit: non-linear least-square minimization and curve-fitting for python. Astro. Source Code Lib. .

Nott, P. R. \& Brady, J. F. 1994 Pressure-driven flow of suspensions: simulation and theory. J. Fluid Mech. 275, 157-199.

Nott, P. R., Guazzelli, E. \& Pouliquen, O. 2011 The suspension balance model revisited. Phys. Fluids 23 (4), 043304. 
Ozenda, O., Saramito, P. \& Chambon, G. 2018 A new rate-independent tensorial model for suspensions of noncolloidal rigid particles in newtonian fluids. J. Rheol. 62 (4), 889-903.

Pailha, M. \& Pouliquen, O. 2009 A two-phase flow description of the initiation of underwater granular avalanches. J. Fluid Mech. 633, 115-135.

Peters, F., Ghigliotti, G., Gallier, S., Blanc, F., Lemaire, E. \& Lobry, L. 2016 Rheology of non-brownian suspensions of rough frictional particles under shear reversal: A numerical study. J. Rheol. 60 (4), 715-732.

Phan-Thien, N. 1995 Constitutive equation for concentrated suspensions in Newtonian liquids. J. Rheol. 39 (4), 679-695.

Radhakrishnan, K. \& Hindmarsh, A. C. 1993 Description and use of lsode, the livermore solver for ordinary differential equations. Tech. Rep.. L.L.N. Lab., Livermore, C.A. (U.S.A.).

Royer, J. R., Blair, D. L. \& Hudson, S. D. 2016 Rheological signature of frictional interactions in shear thickening suspensions. Phys. Rev. Let. 116 (18), 188301.

SARAmito, P. 2016 Complex fluids: modelling and algorithms. Springer.

Sierou, A. \& Brady, J. F. 2002 Rheology and microstructure in concentrated noncolloidal suspensions. J. Rheol. 46, 1031-1056.

Singh, A., Mari, R., Denn, M. M. \& Morris, J. F. 2018 A constitutive model for simple shear of dense frictional suspensions. J. Rheol. 62 (2), 457-468.

Singh, A. \& NotT, P. R. 2003 Experimental measurements of the normal stresses in sheared stokesian suspensions. J. Fluid Mech. 490, 293-320.

Stickel, J. J., Phillips, R. J. \& Powell, R. L. 2006 A constitutive model for microstructure and total stress in particulate suspensions. J. Rheol. 50 (4), 379-413.

Stickel, J. J., Phillips, R. J. \& Powell, R. L. 2007 Application of a constitutive model for particulate suspensions: Time-dependent viscometric flows. J. Rheol. 51 (6), 1271-1302.

YAPici, K., Powell, R. L. \& Phillips, R. J. 2009 Particle migration and suspension structure in steady and oscillatory plane Poiseuille flow. Phys. Fluids 21 (5), 053302.

Zarraga, I. E., Hill, D. A. \& Leighton JR, D. T. 2000 The characterization of the total stress of concentrated suspensions of noncolloidal spheres in newtonian fluids. J. Rheol. 44 (2), 185-220.

\section{Appendix A. Eigen directions of $\gamma_{e}$}

We prove here that, in stationary simple shear flow, the depletion angle, i.e. the angle between the $x$ axis and the direction of the eigenvector associated to the largest eigenvalue of $\gamma_{e}$, expresses as:

$$
\delta_{1}=\tan \left(2 \theta_{e}\right)
$$

From (3.3a)-(3.3c), eigenvalues of $\gamma_{e}$, denoted as $\lambda_{\min } \leqslant \lambda_{\max }$, are both non-zero and write:

$$
\begin{aligned}
& \lambda_{\min }=\frac{\delta_{1}^{-1}}{1+\delta_{1}^{-2}\left(1-a^{2}\right)}\left(a \delta_{1}^{-1}-\left(\delta_{1}^{-2}+1\right)^{1 / 2}\right) \\
& \lambda_{\max }=\frac{\delta_{1}^{-1}}{1+\delta_{1}^{-2}\left(1-a^{2}\right)}\left(a \delta_{1}^{-1}+\left(\delta_{1}^{-2}+1\right)^{1 / 2}\right)
\end{aligned}
$$

The corresponding eigenvectors are:

$$
\boldsymbol{v}_{\min }=\left[\begin{array}{c}
\delta_{1}^{-1}-\left(\delta_{1}^{-2}+1\right)^{1 / 2} \\
1
\end{array}\right] \quad \boldsymbol{v}_{\max }=\left[\begin{array}{c}
1 \\
\left(\delta_{1}^{-2}+1\right)^{1 / 2}-\delta_{1}^{-1}
\end{array}\right]
$$

Accordingly, we obtain:

$$
\theta_{e}=\operatorname{atan}\left(\left(\delta_{1}^{-2}+1\right)^{1 / 2}-\delta_{1}^{-1}\right)=\frac{\operatorname{atan}\left(\delta_{1}\right)}{2}
$$


which is equivalent to (A 1 ). Observe that $\left.\forall \delta_{1}>0, \theta_{e} \in\right] 0, \pi / 4[$.

Remark that, while the eigenvalues of $\gamma_{e}$ depend on the parameter $a$ of the tensor derivative, the eigenvectors are independent thereof. Hence, all the relations between microstructure orientation and model parameters derived in Ozenda et al. (2018), notably (A 1), remain unchanged in the present model.

\section{Appendix B. Identification of model constants}

The rheological model proposed in this paper involves seven constants to be identified: $a, b, \omega, \bar{\eta}_{e}, \bar{\beta}, \bar{\delta}_{1}, \bar{\delta}_{2}$. Global identification of these parameters with respect to experimental data was achieved by minimising the following cost function:

$$
\begin{aligned}
J & =\frac{w_{a}}{\psi_{2}-\psi_{1}} \int_{\psi_{1}}^{\psi_{2}}\left|\theta_{e}(\psi)-\theta_{e}^{o b s}(\psi)\right|^{2} \mathrm{~d} \psi+\frac{w_{v}}{\psi_{4}-\psi_{3}} \int_{\psi_{3}}^{\psi_{4}}\left|\eta_{a p p}(\psi)-\eta_{a p p}^{o b s}(\psi)\right|^{2} \mathrm{~d} \psi \\
& +\sum_{i=1}^{2} \frac{w_{s, i}}{\psi_{m}} \int_{0}^{\psi_{m}}\left|\alpha_{i}(\psi)-\alpha_{i}^{o b s}(\psi)\right|^{2} \mathrm{~d} \psi \\
& +\sum_{k=1}^{5} \frac{w_{t, k}}{\gamma_{k}} \int_{0}^{\gamma_{k}}\left|\eta_{a p p}\left(\psi_{k}, \gamma\right)-\eta_{a p p, k}^{o b s}(\gamma)\right|^{2} \mathrm{~d} \gamma
\end{aligned}
$$

The function $\theta_{e}^{o b s}(\psi)$ is a piecewise-constant interpolation of steady-state depletion angle measurements reported by Blanc et al. (2013) for $\psi \in\left[\psi_{1}, \psi_{2}\right]$, with $\psi_{1}=0.088$ and $\psi_{2}=0.98$ the range of reduced volume fraction explored in the experiments. The function $\eta_{a p p}^{o b s}(\psi)$ is a piecewise-constant interpolation of steady-state values of apparent viscosity measured by Blanc et al. (2011) for $\psi \in\left[\psi_{3}, \psi_{4}\right]$, with $\psi_{3}=0.56$ and $\psi_{4}=0.93$ in this case. The functions $\alpha_{1}^{o b s}(\psi)$ and $\alpha_{2}^{o b s}(\psi)$ are piecewise-constant interpolations of steady-state values of normal stress ratios measured by (Couturier et al. 2011) and (Dai et al. 2013) for $\psi \in\left[0, \psi_{m}\right]$, with $\psi_{m}=0.81$ the maximal reduced volume fraction reached in the experiments. The functions $\eta_{a p p, k}^{o b s}(\gamma), k=1 \ldots 5$, represent the transient evolutions of apparent viscosity during the five shear-reversal experiments of Blanc et al. (2011) characterised by reduced volume fractions $\psi_{k}$. Here, $\gamma_{k}, k=1 \ldots 5$, represent the maximal strain reached in each experiment. Lastly, the function $\eta_{a p p}(\psi, \gamma)$ is associated to the transient solution of the model, while the functions $\theta_{e}(\psi), \eta_{a p p}(\psi), \alpha_{1}(\psi)$, and $\alpha_{2}(\psi)$ are associated to the steady-state solution. The factors $w_{a}, w_{e}, w_{s, i}$ and $w_{t, k}$ are the relative weights of the different terms of the cost function $J$.

The minimization problem of $J$ was solved with a Levenberg-Marquardt method (More et al. 1980), using the optimisation library lmfit (Newville et al. 2016). As explained in $\S 4$, larger values had to be used for the weights $w_{a}, w_{e}, w_{s, i}$, with respect to $w_{t, k}$, in order to promote the agreement with steady-state measurements. In addition, after the global identification step, a secondary, one-by-one, adjustment of each model constants was performed in order to further improve the solution. Final values of identified model constants are indicated in table 1. 